\title{
The Role of Heated Intraperitoneal Chemotherapy (HIPEC) in Ovarian Cancer: Hope or Hoax?
}

\author{
Thomas J. Herzog, MD, FACS \\ Division of Gynecologic Oncology, Department of Obstetrics \& Gynecology, Columbia University College of Physicians \\ \& Surgeons, New York, NY
}

\section{BACKGROUND}

The role of heated intraperitoneal chemotherapy (HIPEC) to treat peritoneal-based cancers has been debated for years. Meanwhile papers that advocate the advantages of this modality continue to accrue, but questions regarding efficacy, optimal indications, patient selection, and toxicity remain largely unresolved. This uncertainty for HIPEC benefit is especially acute in ovarian cancer. Is it time for clinicians to incorporate this treatment modality into the care of ovarian cancer patients? If so, then in whom should this aggressive therapy be prescribed, front-line or recurrent disease? In examining the use of this modality, one needs to consider the defining elements that constitute HIPEC therapy.

\section{THE SURGERY: AGGRESSIVE CYTOREDUCTION}

Generally, the initial aspect of care is to achieve an optimal surgical cytoreduction. Our understanding of the value of cytoreduction in ovarian cancer has morphed over the past several decades to a current definition that ideally results in no residual disease. Previously, the definitions of optimal cytoreduction referenced residual tumors of less than $2 \mathrm{~cm}$ and then less than $1 \mathrm{~cm}$ in greatest diameter. Most experts who treat ovarian cancer embrace the concept of optimal cytoreduction as a critical component of treatment especially in front-line therapy. Many also advocate the value of secondary cytoreduction in appropriately selected patients in the recurrent setting whereby the treatment-free interval is long (generally $>12$ months), and

(C) Society of Surgical Oncology 2012

Published Online: 26 July 2012

T. J. Herzog, MD, FACS

e-mail: th2135@columbia.edu the tumor is completely resectable. Many interventions including peritoneal stripping, diaphragmatic resection, splenectomy, partial hepatectomy, and bowel resections embody aggressive surgical cytoreduction. The surgical aspects of HIPEC protocols are the least controversial component in lieu of a plethora of data, albeit nonrandomized, that demonstrates improved survival with optimal cytoreduction.

\section{ROUTE OF CHEMOTHERAPY ADMINISTRATION: INTRAPERITONEAL THERAPY}

While debated by some, most experts do agree that intraperitoneal (IP) chemotherapy results in a survival advantage for patients especially in the front-line setting. The phase III trials and meta-analyses have demonstrated a statistically significant improvement in progression-free survival (PFS) and overall survival (OS). ${ }^{1}$

\section{THERMAL EFFECT: HEATING THE CHEMOTHERAPY}

The theory behind heating chemotherapy has generated significant interest as a number of in vitro and animal models have demonstrated improved cytotoxicity. The heated chemotherapy is administrated at range of 41- $46{ }^{\circ} \mathrm{C}$. Some studies have done repeated courses of heated chemotherapy with laparoscopy or recatheterization. Most studies do single bathing of the peritoneal cavity. The theories as to the benefit of heating the chemotherapy are numerous and include improved tumor penetration, increased platinum adducts, and possibly immune or cell cycle effects. The heating and infusion adds 1-2 plus hours to the surgical procedure and can be done using either open or closed techniques. ${ }^{2}$ 


\section{THE STUDY IN QUESTION}

In this issue, Bakrin et al. report on 246 patients treated over a 17 -year period who underwent optimal cytoreductive surgery and HIPEC. ${ }^{3}$ Optimal surgical debulking was achieved in $92 \%$ of the patients. Mortality and morbidity (grade 3/4) rates were 0.37 and $12 \%$, respectively. Anastomotic leak occurred in $5 \%$ of patients. The overall median survival was 48.9 months. A total of 62 patients were platinum resistant, and 184 were defined as platinum sensitive. No difference in survival was observed for those who were platinum resistant versus platinum sensitive. Median diseasefree survival was 12.8 months, and the disease-free survival rates at 1,3 , and 5 years were 50,20 , and $9 \%$, respectively.

The strengths of this study are that the authors accrued a large cohort, and the trial was multicentered. Also, the subject matter is clinically relevant, as the role of HIPEC in ovarian cancer is not well defined. The investigators achieved an impressively high optimal cytoreductive status that exceeds most centers for even primary surgery. They described relevant corollary data such as peritoneal cancer indices and cancer resection scores that correlated with outcomes.

The weaknesses are that the study was nonrandomized with no comparator arm. They choose to include both platinum-resistant and platinum-sensitive patients, thus resulting in a heterogeneous group. Furthermore, the patient population may be highly selected especially in lieu of the very high optimal cytoreductive status reported. The 17-year enrollment period also makes data comparison to contemporary treatment strategies problematic. Also troubling is the fact that the authors did not observe any difference in survival for those with platinum-resistant versus platinum-sensitive disease. One would expect HIPEC to benefit both groups, and most would predict a preferential benefit to platinum-sensitive patients. Either the authors have discovered a new paradigm whereby the magnitude of positive effect from HIPEC preferentially benefits platinum-resistant patients or some alternative dynamics peculiar to this study were potentially operative.

\section{PUTTING THIS STUDY INTO PERSPECTIVE}

Currently, there are more than 40 studies from more than ten countries that have reported their data on the role of HIPEC in ovarian cancer. Unfortunately, most of these trials are small in number $(<50$ patients) and have broad entry criteria. The confidence intervals of the results in most cases approach the outcomes achieved in highly selected patients who receive optimal front-line cytoreduction with IP chemotherapy (median overall survival $>5$ years) or secondary cytoreduction in recurrent disease with contemporary chemotherapy regimens (median overall survival $>3$ years).

Toxicity from HIPEC has been variably reported with wide ranges observed. Mortality has been $0-3 \%$ of patients, and major morbidity has been reported in 3-50\% of HIPEC cases. ${ }^{4}$ The relatively high rate of anastomotic leak seen in this issues' study and others are concerning. Separating the adverse events of HIPEC from the inherent risks of aggressive cytoreduction is an important distinction for future trials.

So who is most likely to benefit? Again the literature is severely lacking as trials have reported the use of HIPEC throughout the natural history of ovarian cancer, but most of these studies have overly substratified these groups so as to limit statistical power. One review has advocated a greater benefit for HIPEC with secondary cytoreduction for advanced stage front-line treatment and palliative cytoreduction, while less benefit was proposed for early-stage tumors, initial cytoreduction as well as at interval or consolidative cytoreduction. ${ }^{5}$

\section{THE FUTURE WHERE DO WE GO FROM HERE?}

An unfortunate realization in reviewing the HIPEC literature is that well over 500 women with ovarian cancer

TABLE 1 Current randomized phase III ovarian HIPEC trials

\begin{tabular}{|c|c|c|c|c|c|}
\hline Study & PI & Population & $\begin{array}{l}\text { Accrual } \\
\text { goal }\end{array}$ & $\begin{array}{l}\text { Anticipated } \\
\text { completion } \\
\text { (month/year) }\end{array}$ & Study ID number \\
\hline Interval debulk $+/-$ HIPEC & WJ van Driel & $\begin{array}{l}\text { Front-line St III } \\
\text { Interval cyto }\end{array}$ & 280 & $12 / 13$ & NCT00426257 \\
\hline $\begin{array}{l}\text { Second debulk +/- HIPEC } \\
\text { CHIPOR study }\end{array}$ & JM Classe & $\begin{array}{l}\text { Recurrent } 1 \text { st relapse } \\
\text { Responding to } \\
\text { second line }\end{array}$ & 444 & $12 / 18$ & NCT01376752 \\
\hline Surgery $+/-$ HIPEC & G Scambia & $\begin{array}{l}\text { Recurrent } \\
\text { Plat-sensitive }\end{array}$ & 158 & $2 / 15$ & NCT01539785 \\
\hline HIPEC vs. conventional & S-Y Park & Advanced ovarian front-line & 168 & $12 / 15$ & NCT01091636 \\
\hline
\end{tabular}

PI principle investigator 
have been treated with HIPEC, and yet there is no level one data to definitively support its use, as toxicity is likely increased and efficacy has not been proven to exceed contemporary management. While continued investigation is warranted, it is imperative that comparator arms are a constitutive part of any future studies. HIPEC feasibility with "promising" efficacy and "acceptable" morbidity has been shown in myriad of phase I and phase II trials. It is time for randomized phase III trials with tightly defined populations to elucidate if there is added benefit and in which patients. Table 1 shows current trials that meet at least the criteria of randomization. ${ }^{6}$ Our ovarian cancer patients deserve to know the relative benefits and risks of this interesting but unproven treatment modality.

\section{REFERENCES}

1. Hess LM, Benham-Hutchins M, Herzog TJ, Hsu CH, Malone DC, Skrepnek GH, et al. A meta-analysis of the efficacy of intraperitoneal cisplatin for the front-line treatment of ovarian cancer. Int $J$ Gynecol Cancer. 2007;17:561-70.
2. Helm CW. The role of hyperthermic intraperitoneal chemotherapy (HIPEC) in ovarian cancer. Oncologist. 2009;14:683-94.

3. Bakrin N, Cotte E, Golfier F, Gilly FN, Freyer G, Helm W, et al. Cytoreductive surgery and hyperthermic intraperitoneal chemotherapy (HIPEC) for persistent and recurrent advanced ovarian carcinoma: a multicenter prospective study of 246 patients. Ann Surg Oncol. doi:10.1245/s10434-012-2510-4.

4. Dovern E, de Hingh IH, Verwaal VJ, van Driel WJ, Nienhuijs SW. Hyperthermic intraperitoneal chemotherapy added to the treatment of ovarian cancer. A review of achieved results and complications. Eur J Gynaecol Oncol. 2010;31:256-61.

5. Mulier S, Claes JP, Dierieck V, Amiel JO, Pahaut JP, Marcelis L, et al. Survival benefit of adding hyperthermic intraperitoneal chemotherapy (HIPEC) at the different time-points of treatment of ovarian cancer: review of evidence. Curr Pharm Des. 2012; May 14 [Epub ahead of print].

6. ClinicalTrials.gov. 2012. http://clinicaltrials.gov/. Accessed 6 June 2012. 